\title{
High-resolution spectroscopy of two-dimensional electron systems
}

\author{
O. E. Dial, R. C. Ashoori, L. N. Pfeiffer ${ }^{\dagger}$ K. W. West ${ }^{\dagger}$
}

October 24,2018

\begin{abstract}
Spectroscopic methods involving the sudden injection or ejection of electrons in materials are a powerful probe of electronic structure and interactions ${ }^{1}$. These techniques, such as photoemission and tunneling, yield measurements of the "single particle" density of states (SPDOS) spectrum of a system ${ }^{2}$. The SPDOS is proportional to the probability of successfully injecting or ejecting an electron in these experiments. It is equal to the number of electronic states in the system able to accept an injected electron as a function of its energy and is among the most fundamental and directly calculable quantities in theories of highly interacting systems ${ }^{3}$. However, the two-dimensional electron system (2DES), host to remarkable correlated electron states such as the fractional quantum Hall effect ${ }^{4}$, has proven difficult to probe spectroscopically. Here we present an improved version of time domain capacitance spectroscopy (TDCS) ${ }^{\sqrt{5}}$ that now allows us to measure the SPDOS of a 2DES with unprecedented fidelity and resolution. Using TDCS, we perform measurements of a cold 2DES, providing the first direct measurements of the single-particle exchange-enhanced spin gap ${ }^{\sqrt{6}}$ and single particle lifetimes ${ }^{7}$ in the quantum Hall system, as well as the first observations of exchange splitting of Landau levels not at the Fermi surface. The measurements reveal the difficult to reach and beautiful structure present in this highly correlated system far from the Fermi surface.
\end{abstract}

Spectroscopy of the 2DES has been performed optically and using electron tunneling. Traditional optical methods probe long-wavelength collective excitations rather than the SPDOS 8 . Attempts to measure the SPDOS optically have relied on the introduction of local impurities, altering the measured spectrum ${ }^{9}$. Approaches based on tunneling spectroscopy have been hampered by several difficulties $10[1112$. Typically, the tunnel current enters the 2DES perpendicularly to the plane through a tunnel barrier. In steady-state measurements, maintenance of this current requires it to have a path to exit the 2DES. The current can either flow in the plane of the 2DES to a distant contact, or it can tunnel out perpendicularly from the other side of the plane. However, if the in-plane conductivity of the device is small (as frequently occurs in quantum Hall systems), the current cannot flow out through the plane of the 2DES. If the current tunnels out perpendicularly through a second barrier, the two barriers form a resistive divider and variations in the tunnel current alter the 2D density and tunnel voltage. Finally, ohmic heating limits the useful range of both these techniques to small tunnel voltages.

TDCS eliminates all of the major problems associated with 2D tunneling spectroscopy by abandoning steady-state measurement. In TDCS, we replace the ohmic contact to the 2DES with a

* Massachusetts Institute of Technology, Cambridge, MA 02139, USA

$\dagger$ 'Bell Laboratories, Alcatel-Lucent, Murray Hill, New Jersey 07974, USA 
distant, electrically isolated electrode parallel to the plane of the 2DES. We capacitively apply the tunnel voltage using the isolated electrode and then capacitively detect the tunneled electrons rather than removing them through a second contact or barrier. Because the electron does not need to move in the plane of the 2DES to be detected, we can measure the SPDOS even when the $2 \mathrm{DES}$ is insulating or empty. Although this capacitive coupling requires us to use a pulsed AC measurement to extract I-V characteristics, it also allows us to use very short duty cycles (typically $0.01 \%)$ to minimize heating.

In our measurements, the 2DES is sandwiched between two electrodes of a "tunnel capacitor" created from a GaAs heterostructure (Figure 1 $1 \mathrm{a}, \mathrm{b}$ ). The tunneling electrode, used as a source for injected electrons, is separated from the $2 \mathrm{DES}$ by a thin $\mathrm{Al}_{.33} \mathrm{Ga}_{.67} \mathrm{As}$ tunneling barrier. The isolated electrode, used to apply voltages and detect charges, is separated by a thicker, insulating barrier. To minimize scattering, there is no intentional doping in either barrier. The $2 \mathrm{D}$ electron density is controlled from 0 up to $4 \times 10^{11} / \mathrm{cm}^{2}$ by varying the DC voltage applied across the electrodes.

To perform our measurement, we apply a sudden voltage step $V_{\mathrm{P}}(\lesssim 1 \mathrm{~ns}$ rise time), disequilibrating the tunneling electrode from the 2DES. In the absence of bridge compensation, charging the geometric capacitance $(\sim 10 \mathrm{pF})$ between the electrodes would generate a large charge step at the detector. We remove this step by applying a pulse of opposite polarity to a standard capacitor $\mathrm{C}_{\text {std }}$ connected to the isolated electrode (Figure 1p). As electrons tunnel across the barrier, we measure a long slow rise in the total charge on the isolated electrode due to their image charge. Because electrons tunnel only a short distance into the tunnel capacitor to reach the 2DES, this charging signal is much smaller $(<33 \%)$ than the uncompensated initial charge step. The charging signal is measured using a cryogenic amplifier and massive $(250,000 \mathrm{x})$ repetitive signal averaging. We fit the derivative of this signal as a function of time to determine the tunneling current (Figure 1 1 d). In the first instant after the step, the voltage $\mathrm{V}$ between the 2DES and the tunneling electrode is precisely $\mathrm{V}_{\mathrm{P}}$ multiplied by an easily calibrated geometric lever-arm. This, together with the measured tunneling current, gives us a single point on an I-V curve; varying the pulse amplitude traces out the entire curve (Figure 1 1 ).

With continuous application of the pulsed voltage, the 2DES and electrode would re-equilibrate in a few milliseconds. However, we use only the first $\sim 100 \mathrm{~ns}$ of the step to determine the current, before the voltage across the tunnel barrier has relaxed significantly. This short time interval means that little charge is transferred, so electrons are tunneling into and out of cold electronic systems. After this brief measurement window has elapsed, the voltage step is removed in preparation for the next pulse, and the system is allowed to re-equilibrate.

Improving on our prior work ${ }^{5}$, with precise control over the shape of the applied pulses, we are now able to reduce the systematic and random errors in this I-V curve enough to numerically differentiate it, giving $\mathrm{dI} / \mathrm{dV}$, a quantity proportional to the SPDOS multiplied by a slowly varying tunneling matrix element (see supplement). Although the energy resolution is fundamentally limited only by temperature (see the inset of Figure 4 a ), the colorscale plots are taken with lower resolution to reduce acquisition time.

Figure 2 shows typical plots of the SPDOS as a function of energy and Landau level filling fraction $\nu$. We extract the value of the geometric lever-arm by measuring the known cyclotron splitting of the empty well. We estimate the maximum error that accrues in the energy calibration (the error in the $y$-axis) as we move away from zero density and energy to be about $1 \%$, predominately due to variation of the lever-arm from polarization of the 2DES and depletion of the electrodes.

From left to right within each plot, the quantum well starts fully depleted, with Landau levels 
visible above the Fermi energy $(E>0)$. As the well begins to fill, a magnetic field induced Coulomb gap 5 [14]15|16, appearing as a dark band across $\mathrm{E}=0$ in each plot, opens at the Fermi surface. Each Landau level is pinned by its high density of states as it crosses the Fermi energy. This results in a stair-step pattern of successive flat pinned regions followed by downward steps corresponding to chemical potential jumps as the next Landau level is pulled down to the Fermi energy 17 .

Exchange-enhanced spin-splitting is visible as splitting of the Landau levels as they cross the Fermi surface. At even-integer filling, there is no net spin polarization, causing the exchange gap to vanish. As the density is raised, one spin state of the orbital Landau level begins to fill, and the attractive exchange interaction creates a gap to the other spin state of the orbital level that grows until it saturates with one completely filled spin state at odd-integer $\nu$. The gap shrinks again as the upper spin state is filled and pulled down in energy to meet the lower spin state. This gives rise to the "oscillatory g-factor" in quantum Hall systems ${ }^{6}$ and, together with the pinning of the spin state being filled to the Fermi energy, creates a distinctive pattern as each Landau level crosses the Fermi surface.

We measure the spin-splittings, $E_{j}$, at odd-integer filling by fitting the peaks in constant $\nu$ linecuts of the spectra to Lorentzians and develop an empirical formula for describing the splittings. The results are shown in Figure 3 a. Varying density at fixed values of the magnetic field, we find that the spin gaps diminish with a $1 / \sqrt{\nu}$ dependence. To compare the B-field dependence of splittings at different $\nu$, Figure 3 c shows the splittings multiplied by $\sqrt{\nu}$ as a function of B-field. The data cleanly collapse onto a single line, revealing a B-linear dependence. The splittings are well characterized by $E_{j}=1.2 \pm 0.03 \mathrm{~B} / \sqrt{\nu} \mathrm{meV} / \mathrm{T}$ (Figure $3 \mathrm{~b}$ ), where we have forced the fit through the origin.

No previous experiment has directly measured the single-particle exchange gap that arises in the sudden introduction of a single electron. However, an exchange enhanced gap is also present in the "thermodynamic" density of states often measured using capacitance measurements. These measurements monitor the change in the 2DES chemical potential long after adding electrons to the system, allowing time for correlations to form $13[18$.

Nonetheless, a B-linear dependence has also been seen both in capacitance measurements of the gap in the thermodynamic density of states $\frac{18}{18}$ and in thermally activated measurements of the transport gap 19. However, the measured values of $E_{j}$ are roughly a factor of 4 or 2 smaller than our measurements, respectively. In contrast with our results, thermal activation experiments find no $\nu$ dependence at fixed field $\mathrm{d}^{19 / 20}$.

In the absence of Landau level mixing, the exchange-enhanced spin-gap at the Fermi energy, $E_{j}$, is calculated ${ }^{21 / 22}$ to grow as $\sqrt{B}$ at fixed $\nu$ and to be largest at $\nu=1$ compared with other odd-integer fillings. However, we expect that mixing is important; for $B<6.5 \mathrm{~T}$, the exchange energy scale $e^{2} / \epsilon l_{b}$ is greater than the cyclotron splitting $\hbar \omega_{c}$, and level repulsion from nearby Landau levels should limit the exchange splitting to $\sim \hbar \omega_{c}$. This B-linear dependence has been found in structure-specific calculations ${ }^{23}$. More recent theory by Iordanski, providing results only at $\nu=1$, predicts a B-linear splitting roughly $30 \%$ smaller than our value 24 . No theory predicts the observed $B / \sqrt{\nu}$ dependence.

We also observe for the first time the indirect exchange splitting of Landau levels above and below the Fermi surface by spin-polarized Landau levels at the Fermi surface (yellow ovals in Figure 2). Calculations of the exchange matrix element ${ }^{211}$ between different orbital Landau levels indicate that because of smaller wavefunction overlap, at any given density this indirect splitting should be smaller than the exchange-enhanced spin gap at the Fermi energy (neglecting Landau level mixing). While we find that this is sometimes correct, the most deeply buried orbital Landau level $N=0$ is 
consistently split more than any other level in the spectrum. This level is unique in that there is no deeper Landau level available for level mixing, so there is no level repulsion to limit the exchange spitting on the lower energy side. We suggest that level mixing generally reduces these indirect exchange splittings just as it reduces the splitting at the Fermi surface, but in the case of $N=0$ the absence of lower energy Landau levels allows the splitting to grow larger.

The most remarkable aspect of this observation is that the split Landau levels are at energies several hundred times the thermal energy, yet their splitting reflects the relatively delicate exchange splitting at the Fermi surface. This demonstrates that the high energy features in the spectra depend on properties of the many-body system only present at low temperatures. The high energy spectra constitute a new and previously unexplored probe of the highly correlated many-body ground state.

The lineshapes of Landau levels away from the Fermi energy provide a unique window into the effects of electron-electron interactions. We find that the lineshape at fields of 1 Tesla or less fits well to Lorentzians (inset, Figure 4a). With a nearly empty quantum well, sharp Landau levels are observed at any energy (blue box in Figure $4 \mathrm{a}$ ). As the 2DES is filled, the levels far from the Fermi surface suddenly broaden and disappear. In an occupied 2DES, the injected electron can scatter off electrons already present in the Fermi sea. The phase space available for this scattering grows rapidly as the injection energy moves away from the Fermi energy. At large energies, the short lifetime due to electron-electron scattering becomes the dominant source of broadening. By comparison, at low energies we find maxima in the peak width at even-integer $\nu$ and minima at odd-integer $\nu$. Here, disorder is the chief source of broadening. As observed, this disorder induced broadening should display maxima at even-integer $\nu$, where the density of states of the 2DES at the Fermi level is small and the 2DES screens poorly. It shows minima elsewhere, where the density of states is high and the 2DES screens disorder well.

In the case of a disorder-free 2DES, the electron lifetime $\tau_{e e}$ has been calculated at zero field by Chaplik (see supplement) ${ }^{7}$. At small magnetic fields such that the cyclotron energy is small compared to the Coulomb interaction energy we expect this result to hold. Fitting the widths of the Landau levels to Lorentzians gives us an irregularly spaced mesh of broadenings as a function of energy and density. In order to simplify comparisons, we fit a smooth function through the observed width of the Landau levels at each density, and plot contours of constant broadening (Figure 4 $\mathrm{b}$ ). At high energies and away from even-integer $\nu$, we see good agreement with Chaplik's result over a broad range of densities with no adjustable parameters; this is the region where disorder broadening is small and we expect lifetime broadening to dominate. Prior measurements of momentum and phase scattering times have found quantitative agreement with Chaplik's result using an adjustable scaling parameter, but these data represent the first direct measurement of the single particle lifetime 25126 .

\section{References}

[1] Giaever, I. Energy gap in superconductors measured by electron tunneling. Phys. Rev. Lett. 5, 147-148 (1960).

[2] Bardeen, J. Tunnelling from a many-particle point of view. Phys. Rev. Lett. 6, 57-59 (1961).

[3] Hedin, L., Lundqvist, B. I. \& Lundqvist, S. Beyond the one-electron approximation: Density of states for interacting electrons. In Electronic Density of States, NBS Special Publication 323, 233-249 (Nat. Bur. Stand., 1971). 
[4] Stormer, H. L., Tsui, D. C. \& Gossard, A. C. The fractional quantum hall effect. Rev. Mod. Phys. 71, S298-S305 (1999).

[5] Chan, H. B., Glicofridis, P. I., Ashoori, R. C. \& Melloch, M. R. Universal linear density of states for tunneling into the two-dimensional electron gas in a magnetic field. Phys. Rev. Lett. 79, 2867-2870 (1997).

[6] Ando, T. \& Uemura, Y. Theory of oscillatory g factor in an MOS inversion layer under strong magnetic fields. J. Phys. Soc. Jpn. 37, 1044-1052 (1974).

[7] Chaplik, A. V. Energy spectrum and electron scattering processes in inversion layers. Sov. Phys. - JETP 33, 997-1000 (1971).

[8] Pinczuk, A. et al. Observation of roton density of states in two-dimensional Landau-level excitations. Phys. Rev. Lett. 61, 2701-2704 (1988).

[9] Kukushkin, I. V. \& Timofeev, V. B. Magneto-optics of strongly correlated two-dimensional electrons in single heterojunctions. Advances in Physics 45, 147-242 (1996).

[10] Mendez, E. E., Esaki, L. \& Wang, W. I. Resonant magnetotunneling in GaAlAs-GaAs-GaAlAs heterostructures. Phys. Rev. B 33, 2893-2896 (1986).

[11] Eisenstein, J. P., Gramila, T. J., Pfeiffer, L. N. \& West, K. W. Probing a two-dimensional Fermi surface by tunneling. Phys. Rev. B 44, 6511-6514 (1991).

[12] Main, P. C. et al. Landau-level spectroscopy of a two-dimensional electron system by tunneling through a quantum dot. Phys. Rev. Lett. 84, 729-732 (2000).

[13] Ashoori, R. The Density of States in the Two-Dimensional Electron Gas and Quantum Dots. Ph.D. thesis, Cornell University, URL http://arxiv.org/abs/cond-mat/0607739 (1991).

[14] Aleiner, I. L., Baranger, H. U. \& Glazman, L. I. Tunneling into a two-dimensional electron liquid in a weak magnetic field. Phys. Rev. Lett. 74, 3435-3438 (1995).

[15] Ashoori, R. C., Lebens, J. A., Bigelow, N. P. \& Silsbee, R. H. Equilibrium tunneling from the 2-dimensional electron-gas in GaAs - evidence for a magnetic-field-induced energy-gap. Physical Review Letters 64, 681-684 (1990).

[16] Eisenstein, J. P., Pfeiffer, L. N. \& West, K. W. Coulomb barrier to tunneling between parallel 2-dimensional electron-systems. Physical Review Letters 69, 3804-3807 (1992).

[17] Popov, V. G. Self-consistent equilibrium of a two-dimensional electron system with a reservoir in a quantizing magnetic field: Analytical approach. Physical Review B (Condensed Matter and Materials Physics) 73, 125310 (2006).

[18] Dolgopolov, V. T. et al. Direct measurements of the spin gap in the two-dimensional electron gas of AlGaAs-GaAs heterojunctions. Phys. Rev. Lett. 79, 729-732 (1997).

[19] Usher, A., Nicholas, R. J., Harris, J. J. \& Foxon, C. T. Observation of magnetic excitons and spin waves in activation studies of a two-dimensional electron gas. Phys. Rev. B 41, 1129-1134 (1990). 
[20] Schmeller, A., Eisenstein, J. P., Pfeiffer, L. N. \& West, K. W. Evidence for skyrmions and single spin flips in the integer quantized Hall effect. Phys. Rev. Lett. 75, 4290-4293 (1995).

[21] MacDonald, A. H., Oji, H. C. A. \& Liu, K. L. Thermodynamic properties of an interacting two-dimensional electron gas in a strong magnetic field. Phys. Rev. B 34, 2681-2689 (1986).

[22] Aleiner, I. L. \& Glazman, L. I. Two-dimensional electron liquid in a weak magnetic field. Phys. Rev. B 52, 11296-11312 (1995).

[23] Smith, A. P., MacDonald, A. H. \& Gumbs, G. Quasiparticle effective mass and enhanced g factor for a two-dimensional electron gas at intermediate magnetic fields. Phys. Rev. B 45, 8829-8832 (1992).

[24] Iordanski, S. V. \& Kasbuba, A. Excitations in a quantum Hall ferromagnet with strong Coulomb interaction. JETP Letters 75, 348-353 (2002).

[25] Murphy, S. Q., Eisenstein, J. P., Pfeiffer, L. N. \& West, K. W. Lifetime of two-dimensional electrons measured by tunneling spectroscopy. Phys. Rev. B 52, 14825-14828 (1995).

[26] Yacoby, A., Sivan, U., Umbach, C. P. \& Hong, J. M. Interference and dephasing by electronelectron interaction on length scales shorter than the elastic mean free path. Phys. Rev. Lett. 66, 1938-1941 (1991).

Acknowledgements This work was supported by Office of Naval Research and by the National Science Foundation funded through the NSEC Program and the MRSEC Program. 


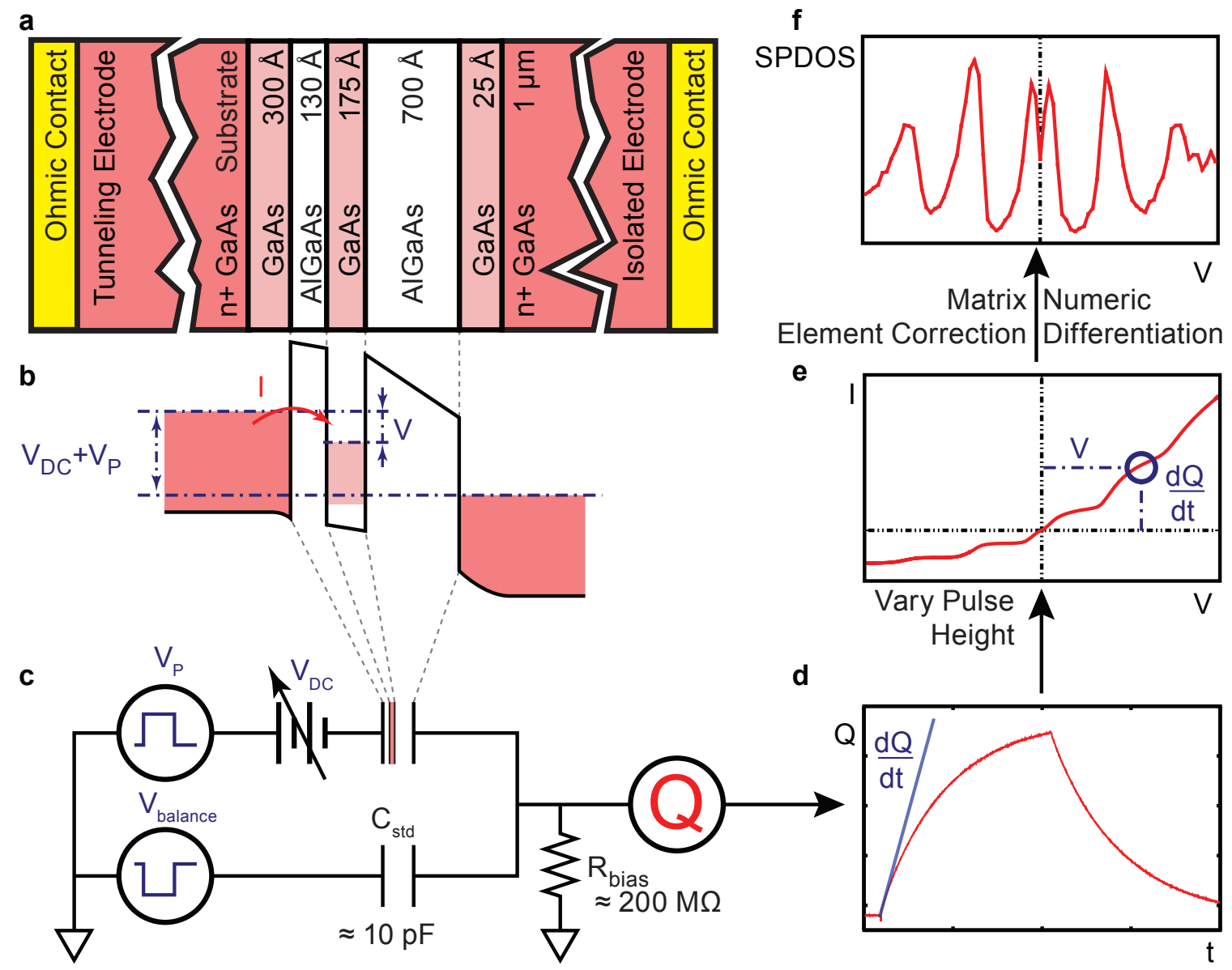

Figure 1: Time domain capacitance spectroscopy. a, GaAs heterostructure used to construct the tunnel capacitor. The only ohmic contacts are to the top and bottom 3D doped regions, used as electrodes. b, Band structure of the tunnel capacitor in the instant after a pulse is applied across it, showing the well defined voltage between the tunneling electrode and 2DES, and the tunnel current. c, Simplified schematic of circuitry used to perform the measurement, including a standard capacitor $\mathrm{C}_{\text {std }}$ used to remove the large charge step from the background geometric capacitance of the structure. d, Measured charge traces, taken with a long pulse for illustrative purposes, showing the charge response averaged over 250,000 repetitions. The slope of this curve immediately after the pulse is applied gives the tunnel current. e, I-V characteristic for tunneling into the 2DES constructed by combining data from $\sim 200$ different applied pulse heights. f, Single particle density of states spectrum attained by multiplying $\mathbf{e}$ by a smooth matrix element correction and differentiating. 


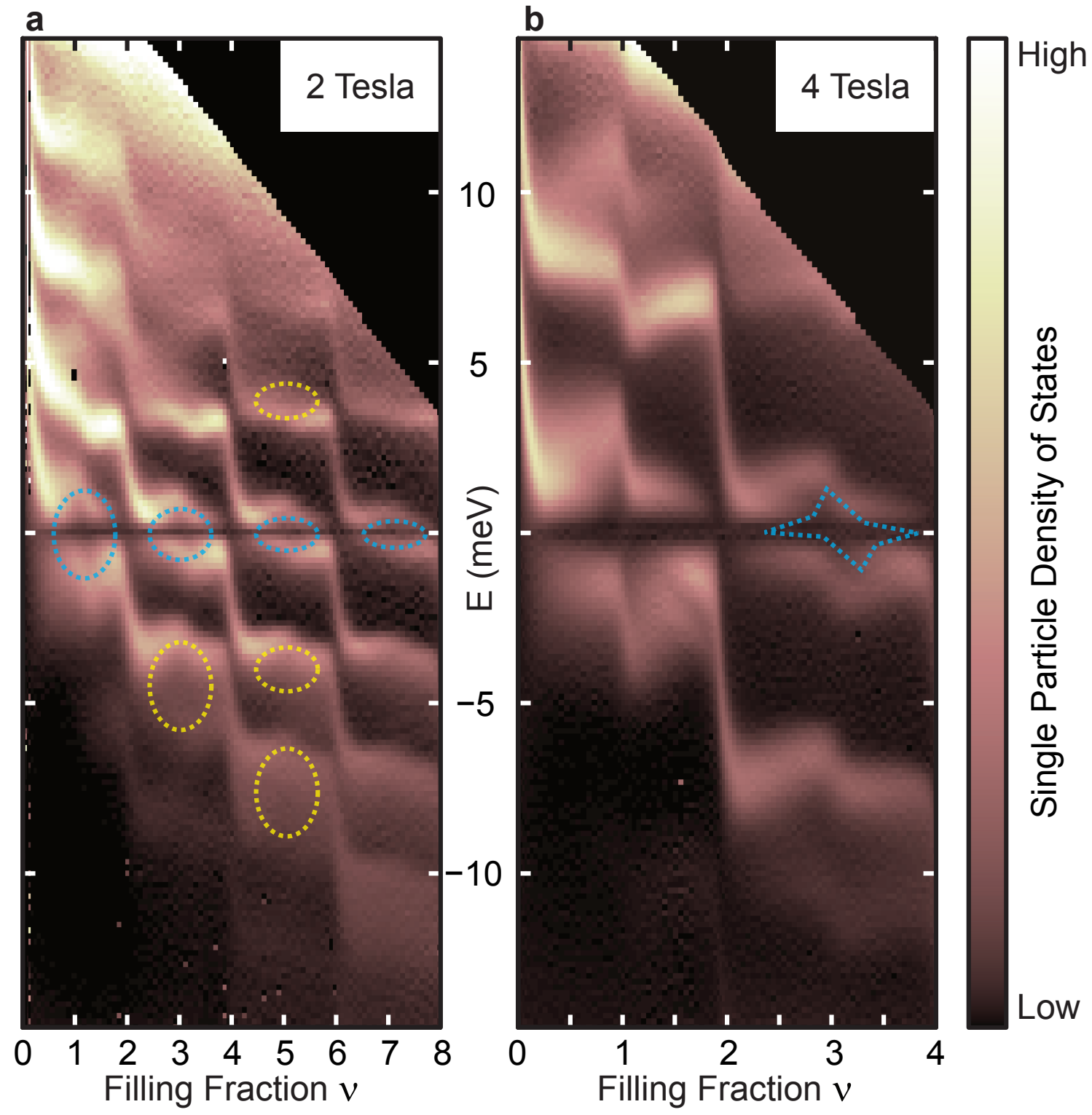

Figure 2: Single particle density of states spectra. The spectra are taken at fixed magnetic fields of 2 and 4 Tesla, with an energy resolution of $170 \mu \mathrm{eV}$ and taken at approximately $100 \mathrm{mK}$. Each bright peak corresponds to a Landau level. The vertical $(y)$ axis is the tunneling energy measured with respect to the Fermi energy. Energies greater than zero correspond to injecting electrons into empty states in the quantum well, while energies less than zero correspond to ejecting electrons from filled states. A smooth approximate correction for variation of the tunneling matrix element has been applied (see supplement), resulting in a dark band in the top-right corner of each plot. The horizontal $(x)$ axis is filling fraction $\nu$ (proportional to electron density at fixed magnetic field). The applied DC gate voltage is transformed to $\nu$ through AC capacitance measurements 13 . Blue ellipses in the $2 \mathrm{~T}$ plot mark exchange enhanced spin splittings, while yellow ellipses mark some of the indirect exchange splittings. The blus dashed shape in the $4 \mathrm{~T}$ plot shows the expected behavior of the Landau levels as they cross the Fermi energy. 

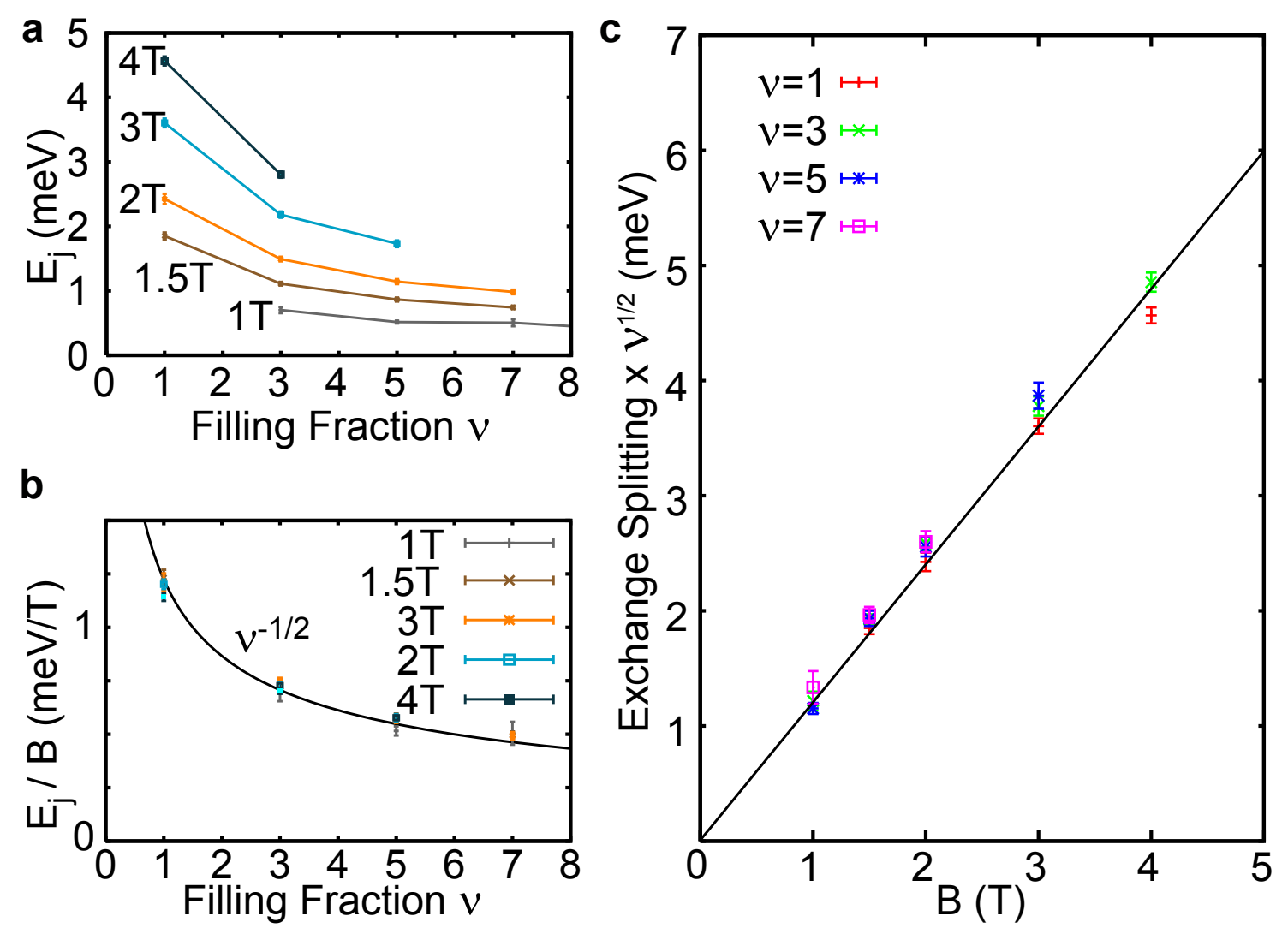

Figure 3: Exchange-enhanced spin splitting at the Fermi energy. a, the unscaled exchangeenhanced spin gap as a function of odd-integer $\nu$ and field. $\mathbf{b}, \nu^{-1 / 2}$ dependence (solid curve) shown by scaling the splitting as a function of $\nu$ by $1 /$ B. c,$E_{j}$ scaled by $\sqrt{\nu}$, showing the proportionality of $E_{j}$ to $\mathrm{B} / \sqrt{\nu}$. The Landau levels are fit by a superposition of Lorentzians to extract the splittings. Error bars show the standard deviation of the exchange splitting, as determined by the variation of the fit residuals. 

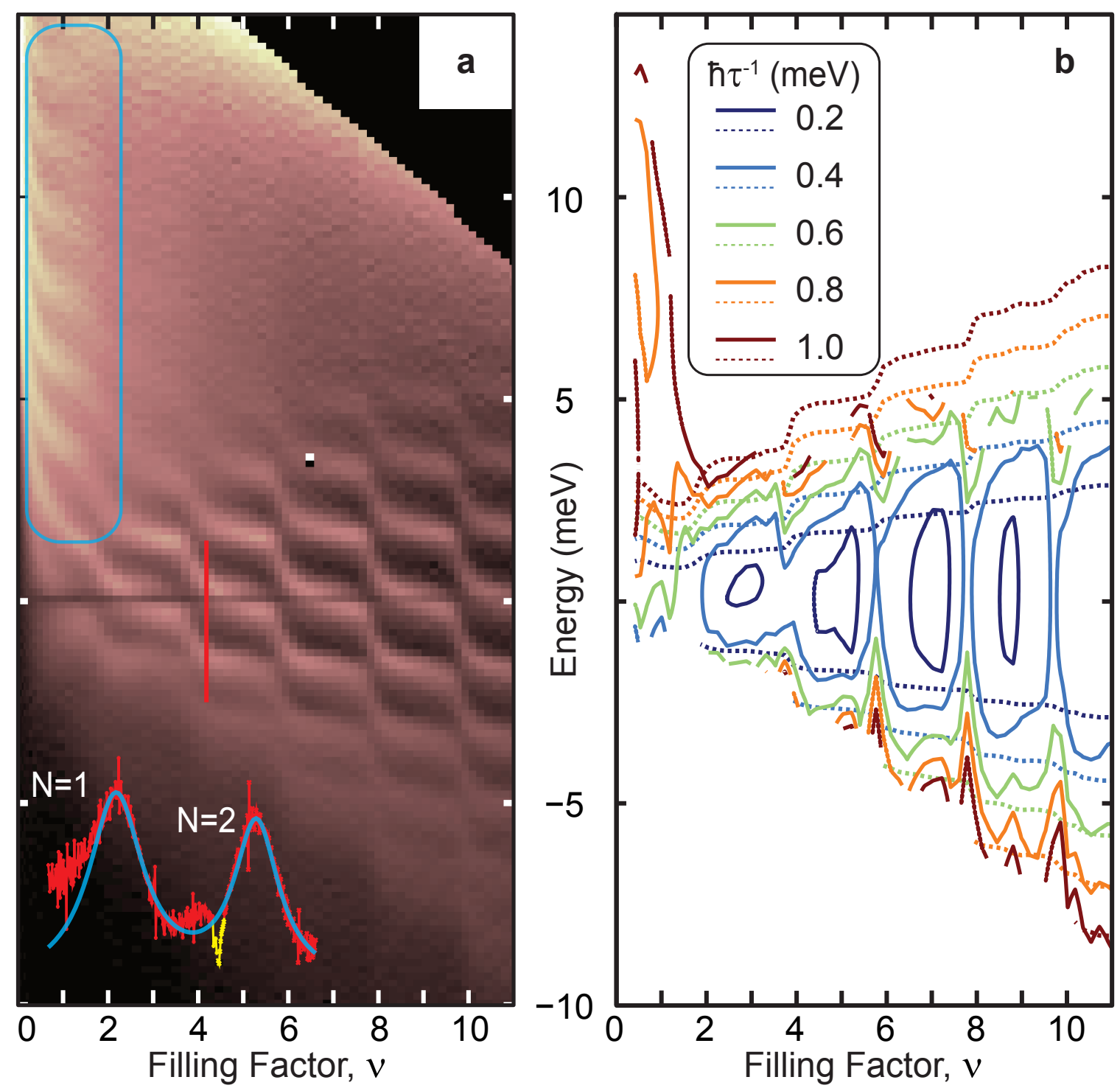

Figure 4: Quasiparticle lifetimes. a, TDCS spectra at 1T. At low densities all Landau levels in the spectrum are resolved (blue box), while at higher densities the short lifetime due to electronelectron interactions broadens the Landau levels farthest from the Fermi surface until they are not resolved. A line cut taken through the red line in the main plot with temperature limited resolution and fit by a superposition of two Lorentzians is shown as an inset. The Coulomb gap is highlighted in yellow in this line cut. Deviations of the fit at large negative energies (left) are from the tail of the $\mathrm{N}=0$ Landau level, not included in the fit. $\mathbf{b}$, Solid lines; contours of constant Landau level HWHM, as obtained by fitting the peaks in a with Lorentzians and interpolating between peaks with a fourth-order polynomial. Dashed lines; $\hbar / \tau_{e e}$ from Chaplik's result ${ }^{7}$ with no adjustable parameters or scaling (see supplement). 


\section{Supplementary Information Matrix element correction}

Here we describe a physically motivated smooth transformation applied to the SPDOS (color scale) of Figures 2 and 4 that results in the normalization of contrast across each spectrum and the removal of an offset from the SPDOS. This transformation only alters the contrast in our spectra and does not change or distort the density $(x)$ or energy $(y)$ axes in any way.

In our experiments, we determine the SPDOS of our 2DES by measuring the tunnel conductivity, $d I / d \mathrm{~V}$. This conductivity can be calculated using Fermi's golden rule ${ }^{27}$ :

$$
I \propto \int_{0}^{e \mathrm{~V}}|M|^{2} \rho_{3 D}(\varepsilon) \rho_{2 D}(\varepsilon) d \varepsilon
$$

Here $\rho_{3 D}$ is the initial SPDOS in our electrode, $\rho_{2 D}$ is the final SPDOS in the 2DES, $M$ is the tunneling matrix element connecting them, and $V$ is the voltage between the 2DES and the tunneling electrode. In many tunneling experiments ${ }^{28}$, measurements are performed over a sufficiently narrow range of energies that $\rho_{3 D}$ and $M$ are approximately constant. In this case $d I / d \mathrm{~V}$ is proportional to $\rho_{2 D}$, the SPDOS. However, because the range of tunneling energies $e \mathrm{~V}$ in our measurement is significant compared to the height of our tunnel barrier and the Fermi energy of our 3D electrode, both $M$ and $\rho_{3 D}$ vary within our spectra. Thus, $d I / d \mathrm{~V}$ is no longer exactly proportional to $\rho_{2 D}$. The changing $M$ and $\rho_{3 D}$ give rise to both a smoothly changing scaling of the SPDOS (color axis) in our spectra and a smoothly changing offset. Both can be seen in the $d I / d \mathrm{~V}$ spectrum in Suppl. Figure 5 a . Because the variation in the scale and offset is small across the width of any given peak in our spectra, they do not measurably affect the observed width or position of any of the Landau levels. While all of the information in the TDCS spectra is present and accessible in the raw $d I / d \mathrm{~V}$ data, even approximate compensation for these variations simplifies visual inspection and fitting of the spectra. Our strategy in performing this compensation is first to approximate $M$ and $\rho_{3 D}$ using first principles calculations and then to "correct" the current to the value it would have if $M$ and $\rho_{3 D}$ were not changing. By applying this correction before differentiating $I$, we remove both the scaling and offset from our spectra. For brevity, we refer to this correction as a "matrix element correction" although it includes terms from both the variation of the tunnel matrix element and the 3D density of states.

We begin by developing an a priori estimate of $M$ and $\rho_{3 D}$ for non-interacting electrons in zero magnetic field. This estimate is a generalization of the approach in [29] to non-equilibrium tunneling. Equating the initial and final energies during tunneling, we have

$$
\frac{\hbar^{2}}{2 m^{*}}\left(k_{2 D, x}^{2}+k_{2 D, y}^{2}\right)+\mathrm{E}_{0}=\frac{\hbar^{2}}{2 m^{*}}\left(k_{3 D, x}^{2}+k_{3 D, y}^{2}+k_{3 D, z}^{2}\right)
$$

Here, $k_{2 D}$ and $k_{3 D}$ are the wavevectors in the 2DES and 3D electrode respectively with the $z$ direction oriented perpendicular to the plane of the electrons in the 2DES. $\mathrm{E}_{0}$ is the energy difference between the $k=0$ states in the 3D and the 2DES, as shown in Suppl. Figure 6. Because our structure has translational symmetry parallel to the quantum well, in-plane momentum is conserved in tunneling 29 . In this case, $k_{2 D, x y}=k_{3 D, x y} \equiv k_{x y}$. Then $k_{3 D, z}=\frac{2 m^{*}}{\hbar^{2}} \sqrt{\mathrm{E}_{0}}$ is determined entirely by the geometry of the structure and the applied voltage, and it is the same for all electrons able to tunnel.

We can represent this condition graphically (see Suppl. Figure 7). First, we plot the 3D Fermi

sphere in $k$ space. We then plot the 2D Fermi disc at $k_{z}=\sqrt{\frac{2 m^{*} \mathrm{E}_{0}}{\hbar^{2}}}$, ensuring that states in the 
2DES are plotted at the same $k_{x}, k_{y}$, and $k_{z}$ as the state in the 3D electrode into which occupying electrons can tunnel while conserving energy and momentum. At equilibrium, Suppl. Figure 7 $\mathrm{a}$, the 2D Fermi disc and the 3D Fermi sphere are filled out to the same circle in $k$ space. Upon disequilibrating the two by applying a pulse, changing $\mathrm{E}_{0}$ and thus the value of $k_{z}$ at which we plot the 2DES, there is a annulus of states which are now occupied in the 2DES but not in the 3D electrode, or vice-versa. These states, depicted in green in Suppl. Figure $7 \mathrm{p}-\mathrm{f}$, contain electrons in either the 2DES or 3D electrode that are able to tunnel.

The 1D $z$ tunneling problem separates from the plane wave states in the $x-y$ plane ${ }^{27}$, so the result only depends on the bound state wavefunction in the $z$ direction and $k_{3 D, z}$ (not on $\left.k_{x y}\right)$; the value of $k_{3 D, z}$ is the same for all electrons able to tunnel. Within a given structure, we can parameterize these using $\mathrm{E}_{0}$. Similarly, because the $x-y$ momentum is fixed by momentum conservation, the 3D density of states $\rho_{3 D}$ is reduced to a $1 \mathrm{D}$ density of states depending only on $k_{3 D, z}$, which in turn only depends on $\mathrm{E}_{0}$. This allows us to move these terms out of the integral in 1. giving

$$
I \propto\left|M\left(\mathrm{E}_{0}\right)\right|^{2} \rho_{3 D}\left(\mathrm{E}_{0}\right) \int_{0}^{e \mathrm{~V}} \rho_{2 D}(\varepsilon) d \varepsilon
$$

Because these terms lie outside the integral, we can correct our tunnel current simply by dividing our measured current by our estimate for $\rho_{3 D}|M|^{2}$. We compute $M$ using the transfer matrix formalism ${ }^{30}$ and the $\mathrm{WKB}^{31}$ approximation. In calculating $M$, there is a leading factor of $k_{3 D, z}$ that arises because electrons with large momenta towards the barrier penetrate more deeply. There are also several smaller corrections from the change in the shape of the barrier and the polarization of the quantum well by the applied voltage. It is convenient to include these by expanding $M$ as $M=k_{3 D, z} M_{0}\left(1-\mathrm{V}_{\mathrm{P}} / \mathrm{V}_{m}\right)$, where $\mathrm{V}_{m}$ is a constant calculated from the first order variation of the tunneling matrix element $M$ and $\mathrm{V}_{\mathrm{P}}$ is our applied pulse height in volts. The remaining $1 \mathrm{D}$ portion of the $3 \mathrm{D}$ density states is proportional to $1 / k_{3 D, z}$, so $\rho_{3 D}|M|^{2}$ is proportional to $k_{3 D, z}\left|M_{0}\right|^{2}\left(1-\mathrm{V}_{\mathrm{P}} / \mathrm{V}_{m}\right)^{2} . k_{3 D, z}$ is proportional to $\sqrt{\left(\mathrm{V}_{\text {align }}-\mathrm{V}_{\mathrm{P}}\right) / \mathrm{V}_{s}}$, where $\mathrm{V}_{\text {align }}$ is the applied voltage pulse that sets $\mathrm{E}_{0}=0$, exactly aligning the $k=0$ states of the $2 \mathrm{D}$ and $3 \mathrm{D}$ bands, and $\mathrm{V}_{s}$ is a scale factor. We then "correct" our current for variation of $M$ and $\rho_{3 D}$ as follows:

$$
I^{\prime}=I \frac{\sqrt{V_{s}}}{\sqrt{\mathrm{V}_{\text {align }}-\mathrm{V}_{\mathrm{P}}}} \frac{1}{\left(1-\mathrm{V}_{\mathrm{P}} / \mathrm{V}_{m}\right)^{2}}
$$

Here $I^{\prime}$ is the corrected current, and $I$ is the measured current. We estimate $\mathrm{V}_{\text {align }}$, which is a function of the 3D Fermi energy and the 2D density, by fitting calculated tunneling I-V curves to our zero field data at two densities and linearly extrapolating to all densities. The constant $\mathrm{V}_{s}$ gives an overall scale to the current and can be neglected, while $\mathrm{V}_{m}$ is a structure-dependent constant, calculated using WKB to be approximately 0.19 Volts for the structure used in this letter.

Because the in-plane momentum is conserved, the tunneling current vanishes if we attempt to inject an electron into a state in the 2DES with a momentum larger than the Fermi momentum in the $3 \mathrm{D}$ electrode. This happens when $\mathrm{V}_{\mathrm{P}}>\mathrm{V}_{\text {align }}$, corresponding to applied voltages slightly larger than that depicted in Suppl. Figure 7f. In this region $\rho_{3 D}$ is zero, and our correction diverges $\left(\sqrt{V_{\text {align }}-V_{\mathrm{P}}}\right.$ in the denominator of Equation 4 goes to zero) because no tunneling can occur that conserves momentum and energy. As we approach this divergence, we are multiplying our experimental data by increasingly large numbers. Well before the correction actually diverges, our SPDOS spectra become dominated by experimental noise. We truncate the data near the point where noise begins to dominate to remove this distraction and substitute a black band. This band 
is visible in the top-right (high density, high energy) corner of the SPDOS spectra in Suppl. Figure 5b and those in Figures 2 and 4. Near these black bands the sharp increase in the corrected SPDOS visible in these figures is an artifact from our rough measurement of $\mathrm{V}_{\text {align }}$ and low order approximation of $M$.

This correction is not exact. Not only is $M$ calculated to low order, but $\mathrm{V}_{\text {align }}$ is only roughly estimated, and we have neglected the effects of interactions. However, the correction does serve to normalize contrast and remove offsets throughout our spectra, simplifying both fitting and visual inspection. Suppl. Figure 5 shows a sample spectrum before and after this matrix element correction; all of the same peaks and features are visible and at the same places; they are simply easier to see after the correction. The observation that the Landau levels all have approximately equal spectral weight after the correction suggests that we have successfully removed the main effects of the tunneling matrix element and $3 \mathrm{D}$ density of states variation from the spectra.

Theoretical electron-electron scattering lifetime

In Figure 4 of the letter, we plot our experimentally extracted line widths against the expected theoretical line width due to electron-electron scattering, as first calculated by Chaplik ${ }^{[32}$. Chaplik's result, also derived by Giuliani and Quinn ${ }^{33}$, is given in terms of quantities used in this letter by:

$$
\frac{\hbar}{\tau_{e e}}(E) \simeq \frac{E_{f}}{4 \pi}\left(\frac{E}{E_{f}}\right)^{2}\left[\ln \left(\frac{E_{f}}{E}\right)+\frac{1}{2}+\ln \left(\frac{2 q_{T F}}{k_{f}}\right)\right]
$$

Here $q_{T F}=2 m e^{2} / \hbar^{2}$ is the Thomas-Fermi screening wave vector in $2 \mathrm{D}, k_{f}$ is the Fermi wavevector, and $E_{f}$ is the Fermi energy. We extract $E_{f}$ from the experimental data by measuring the energy of the lowest $(N=0)$ Landau level relative to the Fermi surface $(E=0$ on the spectrum in Figure $4 \mathrm{a})$, and approximate $k_{f}$ as $\sqrt{2 m E_{f}} / \hbar$. This leaves no free parameters in Chaplik's result, allowing us to make a direct comparison with our data.

\section{References}

[27] Smoliner, J. Tunneling spectroscopy of low-dimensional states. Semicond. Sci. Technol. 11, 1-16 (1996).

[28] Giaever, I. Energy gap in superconductors measured by electron tunneling. Phys. Rev. Lett. 5, 147-148 (1960).

[29] Eisenstein, J. P., Gramila, T. J., Pfeiffer, L. N. \& West, K. W. Probing a two-dimensional Fermi surface by tunneling. Phys. Rev. B 44, 6511-6514 (1991).

[30] Bardeen, J. Tunnelling from a many-particle point of view. Phys. Rev. Lett. 6, 57-59 (1961).

[31] Liboff, R. L. Introductory Quantum Mechanics (Addison-Wesley Publishing Company, 1991), Second edn.

[32] Chaplik, A. V. Energy spectrum and electron scattering processes in inversion layers. Sov. Phys. - JETP 33, 997-1000 (1971).

[33] Giuliani, G. F. \& Quinn, J. J. Lifetime of a quasiparticle in a two-dimensional electron gas. Phys. Rev. B 26, 4421-4428 (1982). 


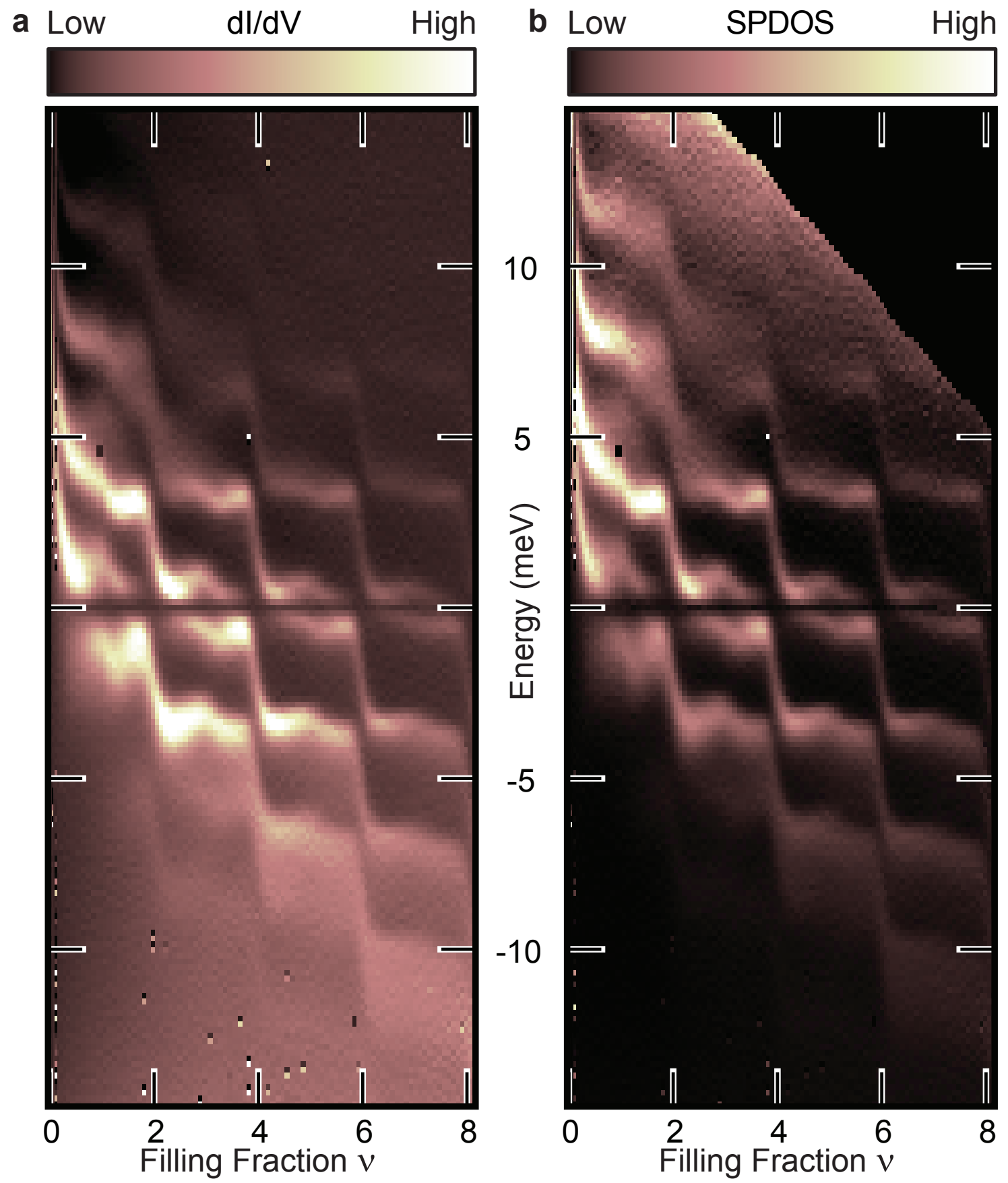

Supplementary Figure 5: $\mathbf{d l} / \mathbf{d V}$ and SPDOS spectra at $\mathbf{2}$ Tesla. Application of the matrix element correction to the $\mathrm{dI} / \mathrm{dV}$ spectra in a yields the SPDOS spectrum shown in $\mathbf{b}$. Note the positions of peaks and peak widths are unchanged, but the corrected version (SPDOS) has more uniform contrast across the peaks and a smaller background. 


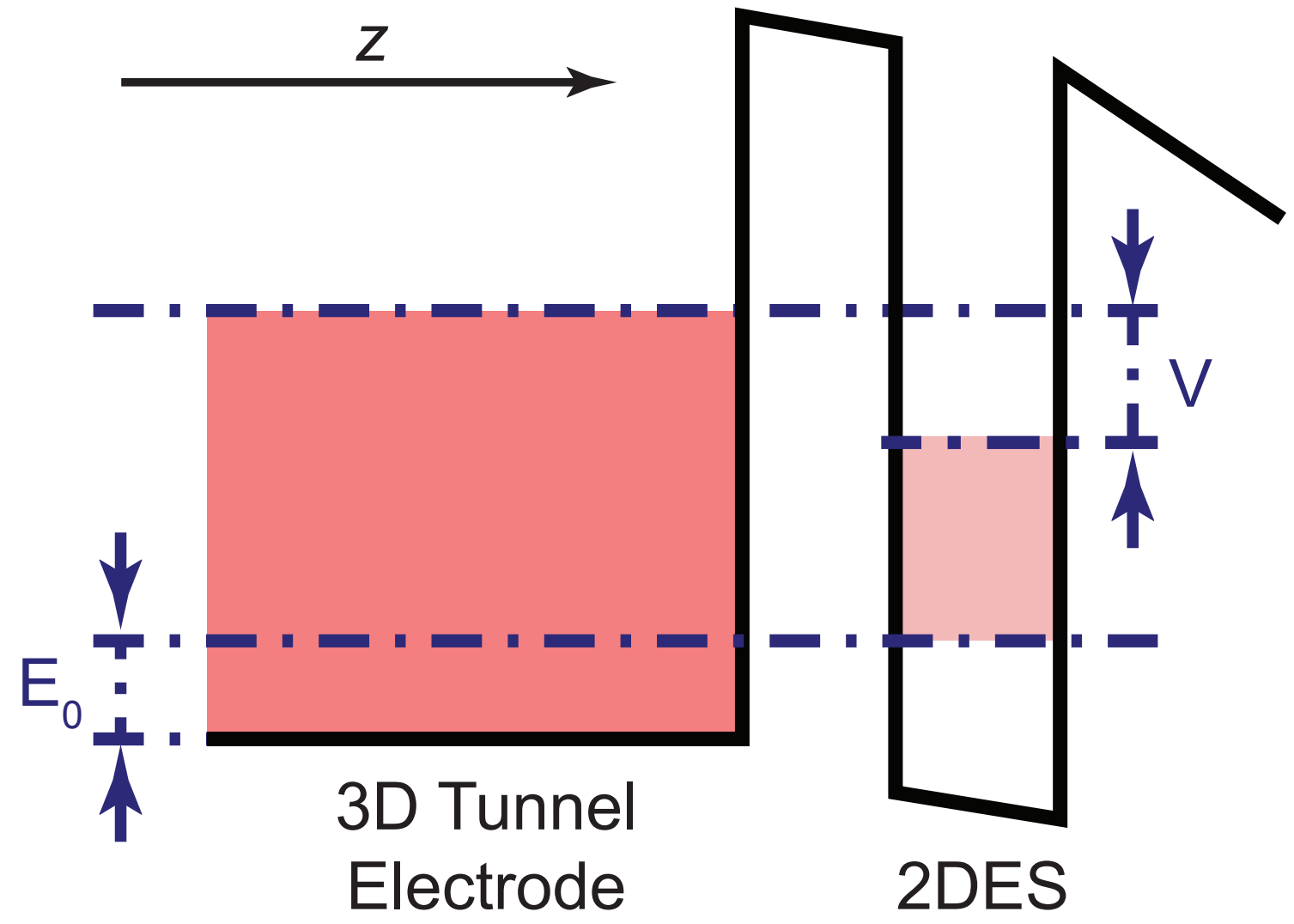

Supplementary Figure 6: Definition of $\mathrm{E}_{0}$. Band diagram of the quantum well and tunneling electrode, showing $\mathrm{E}_{0}$, the energy difference between the quantum well bound state energy and the bottom of the conduction band in the $3 \mathrm{D}$ electrode. $\mathrm{E}_{0}$ depends on any applied AC and DC voltages. 

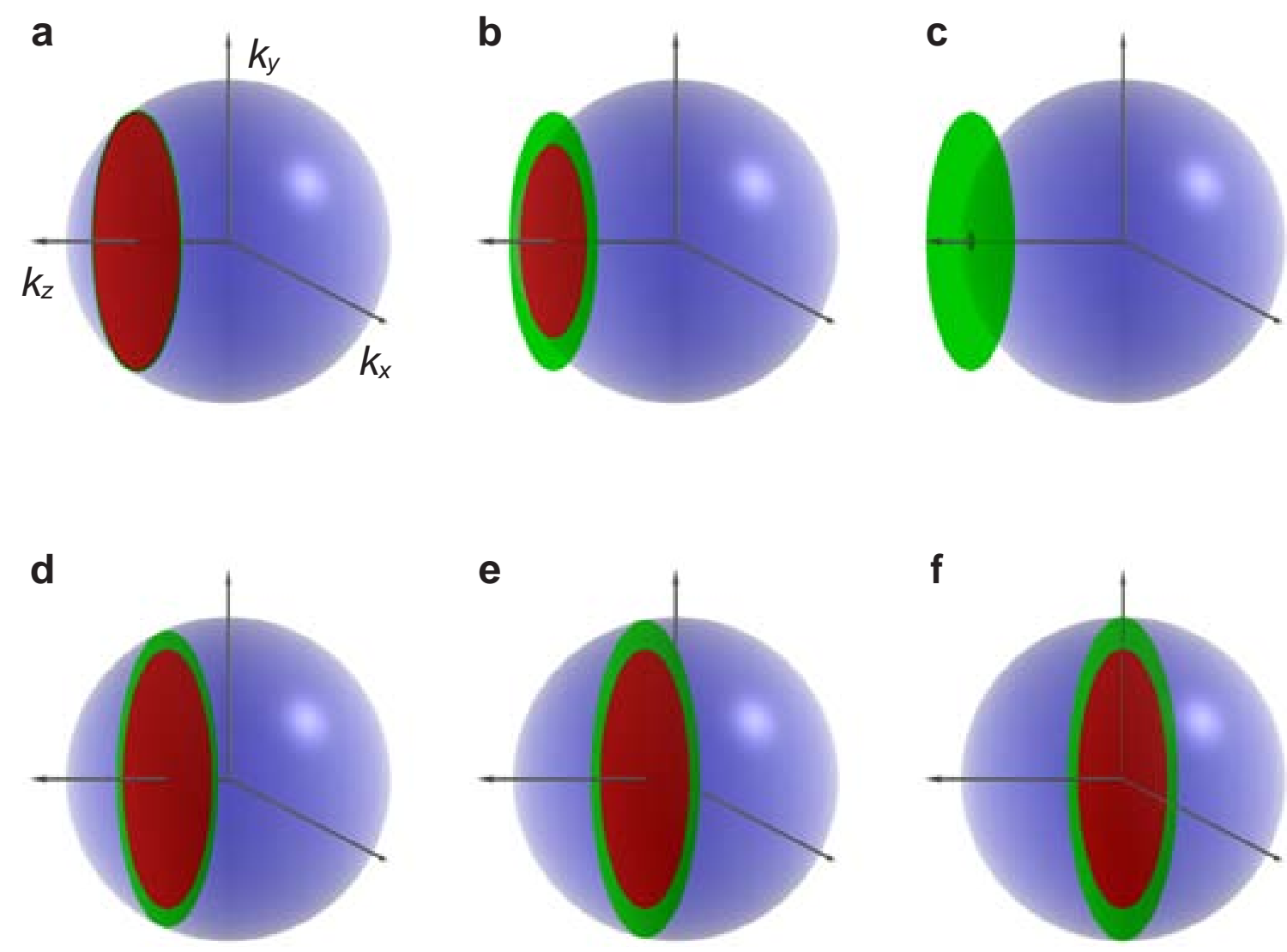

Supplementary Figure 7: Momentum conservation in tunneling. The 3D Fermi sphere and 2D Fermi disc are shown as a function of $k$ vector with a fixed density in the 2DES but with a variety of voltages applied between the 2DES and the Fermi sphere. The Fermi disc has been positioned at the value of $k_{3 D, z}$ into which electrons tunnel from the 2DES while conserving transverse momentum and energy. a, shows the momentum space representation when the 3D Fermi sphere (blue) and 2D Fermi disc (red) are at equilibrium. Only the electrons in the ring of states (green) at the intersection of the Fermi sphere and Fermi disc are able to tunnel. b, shows the Fermi disc and sphere after a pulse has been applied to eject electrons from the 2DES. Electrons in the annulus of filled states in the Fermi disc outside of the Fermi sphere are now able to tunnel. These all tunnel into states with the same $k_{3 D, z}$. c, shows the situation where a sufficiently large pulse has been applied to allow electrons in all the states in the 2DES to tunnel. This situation corresponds to tunneling into the lowest lying (most negative energy) Landau level in our spectra. d-e, show two small injecting pulses, where electrons will be injected into the annulus of states (green) that were once empty in the 2DES but now lie inside the Fermi sphere. $\mathbf{f}$, shows a large injecting voltage. If the injecting voltage is increased any further the tunnel current will be cut off because $V_{P}>V_{\text {align }}$ (see text). This condition corresponds to the beginning of the black bands in the top-right corner of our SPDOS spectra. 